\title{
Quality Control of Storage Phosphor Digital Radiography Systems
}

\author{
Matthew Freedman, Dot Steller, Hamid Jafroudi, and Seong Ki Mun
}

Quality control $(\mathrm{QC})$ of storage phosphor devices is important in assuring that the image information entered into an Image management and communication (IMAC) system is sufficient for diagnosis. QC of storage phosphor digital radiography systems is complex because of the self-corrective nature of the imageprocessing software used in these machines. Currently, one must produce hard copy to perform adequate OC. Inspection of images with reject analysis and inspection of cassettes and imaging plates has helped us in our $\mathrm{QC}$ program. For those $\mathrm{QC}$ tests using control limits, the appropriate settings for these limits are unknown. Starting approximations are given. Recommended tests are described.

Copyright $\mathbb{0} 1995$ by W.B. Saunders Company

KEY WORD: Digital radiography quality.

D IGITAL PROJECTION radiography is an important method for introducing radiographic information into image management and communication (IMAC) systems. There are four methods of digital radiography currently used in medicine and dentistry. These are based on storage phosphors, charge coupled devices, selenium receptors, and image intensifier tubes. This paper will discuss the quality control (QC) of devices using storage phosphor technology based on the author's experience with Fuji and Agfa systems. There have been several prior reports on QC of storage phosphor digital radiography machines. ${ }^{1,2}$ At this time, the authors use hard-copy output for performing QC of storage phosphor projection radiography devices. In this report, the term "computed radiography" (CR) is used to apply to devices using storage phosphor technology.

CR has introduced new requirements for quality control that are different from those needed with other imaging technologies. The balancing of optical density that occurs with $\mathrm{CR}$ systems means that it is more difficult for the radiologist to detect malfunction of the equipment by simple inspection of the image: a more complex system is necessary.

The image processing software of $\mathrm{CR}$ devices results in a robust system designed to internally correct errors in exposure. The mechanisms installed to correct errors in exposure also function quite effectively in minimizing the effect on the final image quality of many internal errors of machine functioning that could occur. Therefore, in clinical practice one tends to see total failure of components rather than progressive deterioration of image quality. The manufacturers of the machines we have tested provide only limited supporting functions for doing $\mathrm{QC}$ of the internal functioning of the machine.

The QC program we use is derived from our 30 months of experience with an Agfa Diagnostic Center (ADC; Mortsel, Belgium) prototype, 49 months of experience with a Fuji (Tokyo, Japan) AC-1 (modified to an AC-1 + with later modification to their high-resolution system) and 10 months experience with a Fuji 9000 . Approximately 100,000 Fuji CR images are now obtained annually. Information was also acquired from manuals provided by Agfa and Fuji and from attendance at the Fuji course used for training their service personnel.

The most frequent errors detected by our QC program are based on human factors rather than machine factors.

In this report, the authors will be describing simple QC procedures that can be used to assess drift from preselected standards of performance. These standards should be first measured after the machine has been initially calibrated by the manufacturer and undergone acceptance testing. A longer document on acceptance testing and $\mathrm{QC}$ is available from the authors. ${ }^{3}$ It is assumed that the readers are well acquainted with the meaning of the imageprocessing factors on the Agfa or Fuji machine they operate. Additional information on optimizing image appearance is available in the literature. ${ }^{4,5}$ This report first discusses the types of failure and then the method of detecting fail-

From the Department of Radiology, Division of Imaging Science and Information Systems, Georgetown University School of Medicine, Washington, $D C$.

Address reprint requests to Matthew Freedman, $M D$, Department of Radiology, Georgetown University Medical Center, 3800 Reservoir Rd, NW, Washington, DC 20007.

Copyright $\odot 1995$ by W.B. Saunders Company 0897-1889/95/0802-0001\$3.00/0 
ures. Suggestions are included for machine design changes in $\mathrm{CR}$ devices that might either decrease the incidence of failures or improve their early detectability.

\section{TYPES OF CR FAILURES}

The purpose of a QC program is to find systematic or generalizable failures early enough so that their effect on overall performance is minimal. The process combines a search for minor deviations from standards that, if continued, would worsen and a review of specific process failures. The difficulties in QC analysis are in differentiating minor deviations that represent trends toward failure from random variations that are self correcting (The data for doing this are currently lacking) and the appropriate generalization from the specific failure to the process that caused the failure.

Patients vary and, therefore, failure of one image may be caused by patient variability or machine failure. Each component of a machine is designed to have a range of tolerance of performance that depends on engineering factors related to the cost of greater perfection. Therefore, a certain amount of variability is expected.

Failures can result from human failure or machine failure. Certain aspects of the design of the CR machine increase the chance of human failure. Certain human factors increase the chance of machine failure.

\section{HUMAN FAILURE AND IMAGE QUALITY OF CR DEVICES}

The most common cause of failed images, in our experience, is technologist error. The three main causes of technologist error are lack of sufficient training in $\mathrm{CR}$, curiosity leading to experimentation, and machine complexity.

\section{Training}

$\mathrm{CR}$ is not a simple replacement device for screen-film radiography. CR introduces several new important concepts that technologists must learn. For example, in $\mathrm{CR}$, resolution is dependent on image plate size; good image quality requires the correct selection of the body part on the identification (ID) station; two images on the same imaging plate (IP) require exposure balancing different than in screen film radiography; and post processing is necessary for some images. Collimation methods can affect image quality on the Fuji AC-1 and Agfa ADC prototype, but, apart from very small field sizes, usually do not affect images on the Fuji 9000. Because the machine corrects for errors in exposure, wide variation in exposure can occur and patients may be exposed to excess radiation. ${ }^{6}$ Many failures result from lack of proper training in this new modality. A prior report ${ }^{6}$ shows the long-term beneficial effect of technologist training.

\section{Curiosity Leading to Experimentation}

Technologists are curious about the equipment they use and are interested in creating the best image possible. Given the opportunity, some will try to figure out how the machine works by trying different settings of the machine parameters, knowing they can usually save a bad image by post-processing on the workstation. Because of a lack of level of access control on the ID terminal, what was intended as a onetime experiment can be loaded into the ID terminal memory as a permanent change affecting many subsequent images. In our initial 3-month experience with the Fuji 9000, we identified unauthorized technologist-induced changes in image-processing settings 12 times. Because of this, we incorporated into our training program hands-on training on how to properly experiment with the system without causing damage. By enlisting the technologists into the search for better imaging with CR, we both decreased the incidence of unauthorized changes (one in the last 5 months) and received several suggestions for improved image quality that we have added to our routine.

In the ninth month, the Fuji 9000 shut down because the memory card was completely filled. The system adds each new image processing setting to its memory rather than overwriting the prior changes. When the memory is full, the system shuts down requiring reloading of software.

Software controls limiting changes in image processing settings to a few key operators would decrease the unauthorized resetting of the factors. 


\section{Machine Complexity}

The operation of these machines is complex and can result in errors. For example, the technologist must enter the body part radiographed into the ID terminal. Technologists often do several examinations on the same patient stacking the cassettes and may not remember which cassette represents which body part. In our multiple image trauma studies, $\sim 10 \%$ of imaging plates will have the body part misidentified. When used for bedside examinations, the lack of a clip on the cassette to hold the patient identification card results in a misidentification rate of $\sim 1$ in 80 images.

When CR is used to enter data into an IMAC system, orientation of the image on the affects whether the final image will be upright; selecting antero-posterior or postero-anterior projection will affect which side the heart is on, etc. The technologist who tries to get a better image through post processing or to correct the effects of misidentification of body parts to create a proper image is limited by lack of knowledge of a complex image-processing system. We have found that hands-on training in image processing allows the technologist to correct most of the errors and increases technologist satisfaction. Machine simplification would be important in decreasing the incidence of technologist error.

\section{MACHINE FAILURE AND DECREASED IMAGE QUALITY IN CR DEVICES}

There are five components of the machine that can result in decreased CR image quality in an IMAC system: the cassette, imaging plate, image plate reader, image processing software, and the image display device. We will not discuss the display device.

\section{Self-Correcting Features of CR Image Quality}

The designers of CR systems have made the systems largely self-corrective to factors that might result in image-quality degradation. The software that corrects for variation in IP exposure by measuring the exposure and then setting a sensitivity or "S" value to control the final image density will also correct variations among imaging plate $\mathrm{x}$-ray absorption, IP light emission, laser power used in the plate reader, deterioration of the light guide and photomultiplier device. It will also correct for certain deterioration in the system electronics. Detecting variations in imaging plates or machine function by monitoring the $S$ value in clinical cases is likely to be masked by variability in exposure settings and patient size. In prior work, we found that well trained and supervised technologists still showed a fivefold variability of S numbers on bedside chest radiographs. ${ }^{6}$

A failing CR system that adjusts its sensitivity to correct for gradual component degradation would introduce more noise into the image (the image would have a lower signal-to-noise ratio). However, the self-corrective nature of the CR machine obscures the visibility of the noise through filtering (on the Fuji systems this is the RT factor). The Agfa ADC prototype provides software for measuring the signal-to-noise ratio; the Fuji software does not.

The self-correcting features for image appearance built into the $\mathrm{CR}$ machines to make them robust for variations in exposure make it quite difficult to detect gradual machine or IP deterioration based on clinical image output. Therefore, tests of machine function must disable these features before the test.

\section{Cassettes}

The cassettes are one of the weak links in CR systems. They need frequent inspection to check on the hinges, ID windows, latches, and lead backing (if lead backed). Failures of each of these have resulted in failures of our Fuji system. One third of our 14- $\times 17$-inch Fuji cassettes placed in the Fuji 9000 failed in the first month because of broken ID windows jamming the machine. The machine jammed once because of detachment of the lead of the lead-backed cassettes. Fuji has redesigned the cassettes. We are testing the recently introduced new model, so far without failures. The Agfa cassettes have not broken.

\section{Imaging Plates}

The IPs develop an electrostatic charge over time that attracts dust. Dust can result in white or black spots. Intense white spots on an image result from reflection of the laser beam more directly into the light guide. Black spots in the 
image result from local failures in the erasure/ activation of the IP: the nonactivated portion of the IP will not record the exposure information on the next exposure. White dots can be confused with microcalcifications in digital mammography and foreign matter in extremity radiographs. (Digital mammography using Fuji CR is in clinical use in Europe and Japan and is in clinical trials in the United States.)

IPs can develop fissures and scratches on their surface. These can be detected on images made from these plates and on direct inspection of the IP surface. These defects cannot be repaired and, if they interfere with diagnosis, the IP should be replaced. On the Fuji system, the ID number of the IP is recorded on the hard-copy image. On the Agfa system, the IP number is matched with the image in the ID station data base. We have had to replace three imaging plates that were damaged in machine jams in the first 3 months of use of the Fuji 9000 and 4 IPs damaged in jams of the AC- $1+$. Initial cracks in the IPs used interchangeably in the Fuji 9000 and Fuji AC-1 started to appear after 5 months of use. We have replaced 14 of the 14- $\times 17$-inch IPs (two thirds of our IPs of this size), two $10-\times 12$-inch IPs, and two $18-\times$ 24-cm IPs for cracks.

We have recently received redesigned IPs for use in our Fuji units. The new design should provide greater mechanical strength.

\section{IP Reader}

The IP reader contains mechanical transport mechanisms, a laser, a light guide, a photomultiplier device, and IP erasure device. Each of these systems can result in image degradation. The transport mechanism can jam and shut down the machine, dust can interfere with the light guide, and the laser, photomultiplier, and erasure light can slowly deteriorate or rapidly fail. Slow deterioration is difficult to detect because of the image self-correction feature of the software. Dust on the light guide can result in streaks or lines on the final image. Laser-scan position accuracy degradation can result in uneven edges of a sharply defined object.

After the initial 3 months of use of the Fuji 9000 in which jams were frequent and usually caused by the cassette problems described above, we now experience approximately one jam every
2 weeks. The machine can jam from IP or cassette transport errors. Light-guide malalignment was detected once on the Fuji AC-1. The erasure light on the Fuji 9000 has failed once resulting in automatic shutdown of the machine.

\section{The Image-Processing Software}

The image-processing software on CR devices is quite complex. Once a machine is calibrated, we have not detected any spontaneous deterioration in it. It is necessary to check that people using the ID terminal have not changed the settings because it is possible to do this inadvertently or as a prank. Three times, our Fuji machine has reverted from our preferred image-processing settings to Fuji's original settings during machine repairs and power outages. We have not had similar problems with the Agfa processing software. A list of the preferred image-processing settings for each body part should be maintained so that if the settings revert, the optimized settings can be reentered. Because this is a particular problem of the Fuji system, it would be helpful if Fuji could provide a memory card to users so they could store their preferred settings and reload them as needed.

\section{ENVIRONMENTAL CAUSES OF DECREASED IMAGE QUALITY}

Dust is one of the main causes of imagequality degradation in CR devices. The IPs, through use, become static charged and avidly attract dust. Control of environmental dust is of high priority.

Users of Fuji CR devices have to be trained to always close the door of the unit between use. It would be helpful if this feature were automatic as it is on the Agfa machine.

The light guide on the Fuji 9000 has required cleaning for dust three times in 9 months; however, it is usually cleaned when the machine case is opened for any purpose. Dust is identified in images requiring special cleaning of IPs approximately three times a week. Because the CR system is a closed system, the cassette is opened only inside the machine and thus the dust accumulation on the IPs should occur only within the machine. Because of the amount of dust accumulating inside our Fuji 9000, Fuji service has recommended that their yearly pre- 
ventive maintenance be performed at 10 months to allow full cleaning of the machine. The air vents on the machine should be cleaned weekly. We usually remove 2 to $4 \mathrm{cc}$ of dust weekly.

Liquids (especially blood and other proteinaceous body fluids) can permanently damage the imaging plates and possibly damage the light guide resulting in expensive repairs. We train our technologists to enclose the cassette in a cover when it might be contaminated with body fluids.

\section{QUALITY CONTROL TEST RECOMMENDATIONS}

There are three methods appropriate for quality control of CR devices: component inspection, reject analysis, and control chart methods. All three methods are important.

\section{Component Inspection}

Cassettes and IPs are both subject to failure. IPs should be cleaned weekly and whenever dirt artifacts are found on an image. This is an appropriate time to inspect the cassettes for partial failures of hinges, ID windows, lead backing, and clasps, and to check the imaging plates for scratches, stains, fissures, and cracks. Bad components should be replaced.

\section{Reject Analysis}

Reject analysis or repeat analysis collects all images that have been replaced with repeat images and attempts to analyze the nature and cause of the failure. Failures may result from unique problems or generic problems. In CR, an analysis of images resulting from repeat exposures or from post processing should both be reviewed. When hard copy is used, the bad images can be kept for later review. When only soft copy is used, a paper or electronic record should be kept of images requiring repeats. Placing the images in an electronic QC folder would simplify their analysis.

Action should be taken based on the reject analysis appropriate to the problem found. The most frequent problems identified by repeat analysis are related to technologist error: improper positioning, improper exposure factors, incorrect selection of body part on the ID station, incorrect selection of imaging plate size or resolution, and incorrect matching of high- resolution IPs with appropriate exposure. The action taken in these cases is technologist education.

Reject analysis resulted in our changing some of our preset image processing to accommodate a wider variety of patient sizes.

\section{Control Limit Tests}

Quality control limit tests are set to measure a factor repeatedly for possible deviations from a standard. Control limits (or action limits) are set so that action can be taken when the measurement deviates beyond a certain amount from the standard. These control limits can be derived in two ways: first, from recording a large number of readings on machines that are functioning appropriately and using two or three standard deviations from the mean as the control limits or, second, by determining the level at which the deviation from the standard results in some measurable important change in function or output or becomes predictive of impending component failure.

Appropriate factors to measure using control limits include $S$ numbers, image optical density, and signal-to-noise ratio. However, currently, setting clinically relevant control limits on these factors is mainly guess work. We have not detected any incipient machine failures that showed variations in these numbers, nor have we seen failures of a type expected to result in variations in these numbers.

\section{Monitoring of the S Number}

The $\mathrm{S}$ number is used on the Fuji systems to indicate the amplification used to correct for differences in exposure. There is no Agfa equivalent.

Monitoring of the $\mathrm{S}$ number in clinical cases may indicate problems caused by $x$-ray machine failures or technologist errors. In clinical cases, $S$ variability is much more likely to be caused by exposure and patient-specific differences rather than machine failure. ${ }^{6}$

Monitoring of $S$ numbers resulting from a standard exposure would detect poor-quality imaging plates, deterioration of imaging plates, decreased laser power, photomultiplier deterioration, malalignment of the light guide, and partial or complete failure of the erasure process. Each of these failures would result in less 
signal reaching the computer for analysis. The computer would correct for these deviations by increasing the $S$ number to create an image of appropriate optical density for the body part.

There are two complementary methods of testing these image acquisition components: use a standard flat-field exposure in auto mode and follow the $\mathrm{S}$ numbers graphically over time, or set a standard $S$ number in the fixed mode and measure the resulting optical densities of the serial images. On the Agfa system, one tests the system by setting the machine in fixed mode and measuring the optical density of the output image on serial images.

\section{Setting the Control Limit on S Numbers}

If one chooses to obtain a standard exposure using standard parameters, one should see a variability of S numbers with SD of about $10 \%$ because that is the manufacturing limit set for the internal control system of the manufacturer. Thus, one can choose to use two or three SDs as the control limit. In making this decision, one would want knowledge of the clinical importance of variations of $S$ numbers of this magnitude and the predictive value of machine failure of $S$ number variability that exceeds that limit. If one is to accept a 60-point spread from an $S$ of 200 , one would want to know whether that magnitude of difference has any clinical significance in diagnosis or any effect on the early detection of machine failure.

Currently we have no information of the predictive value of variations in the $\mathrm{S}$ number as an indicator of incipient machine failure. For research purposes, it would be helpful to compile this data from multiple sites and evaluate its predictive value. We are currently compiling this data on our two Fuji machines.

Is it clinically important to limit variation to 60 units from a mean $S$ of 200 ? Workers in this area would agree that there is some level of exposure below which information is lost. ${ }^{7}$ For chest radiographs (which would normally have an $S$ of 200), an S greater than 500 may result in some loss of information about the locations of tubes in the mediastinum. ${ }^{8}$ In our experience, there is a loss of information about tubes and lines in the mediastinum that occurs between an IP exposure of 0.13 and $0.06 \mathrm{mR}$. It is likely that the $S$ number required for full information will differ with different diagnostic tasks. The required IP exposures for most clinical diagnostic tasks are not known. Loss of information would tend to occur in focal areas of the images receiving the least information. Because the " $S$ " number reflects an average exposure over all regions of the IP and information loss is likely to occur only in those regions of the IP receiving the least exposure, it is difficult to determine the relevance of a change in the global S number to diagnostic accuracy.

At the moment, using control limits to an S of 200 of \pm 60 units seems reasonable, but without much supporting evidence that it is meaningful.

\section{Setting Control Limits for Optical Density (OD)}

As with the $S$ number, there is no evidence that variability of $O D$ in serial images taken with a standard exposure has any predictive value of potential machine failure or serves as an indicator of component degradation.

Variations in (OD) that cause the image to fall more in the toe or shoulder of the contrast curve can affect diagnosis. In the more usual $C R$ operating modes of auto or semi mode, the image processing will automatically correct the optical density so the main effect in most clinical uses of $\mathrm{CR}$ will be that differences in exposure affect the noise in the image, rather than its OD. Thus, the clinical meaning of variations in OD occurring during QC studies is unknown.

\section{Measuring Signal-to-Noise Ratio}

Signal-to-noise measurements can be made on the Agfa workstation, but not on the Fuji machines. Tracking of this measurement would track performance of multiple components: the IP and components of the plate reader. We are unaware of any data on the predictive value of this for predicting failure of components. Values of SNR affecting diagnosis would vary with the image features of the disease in question. We are not aware of current data that would suggest appropriate control limits for this factor.

\section{QC RECOMMENDATIONS}

\section{Initial Control Images}

The machine should be properly calibrated by the manufacturer and acceptance testing completed. Then images should be obtained using available phantoms. One should use a 
well-calibrated $\mathrm{x}$-ray machine that is expected to be available for many years. Variability will be reduced if the same IP is used for both the initial and follow-up tests.

Images of phantoms. For each available phantom (chest, hand, skull, or whatever is available), obtain two images processed with your standard algorithm settings for that body part. These images should be stored for future comparison with new images obtained with the same exposure and image-processing factors. Although we do not recommend routinely repeating these images, they may prove helpful in confirming that a change in image quality detected on the step wedge test (test B) has occurred.

Images of a step wedge. Images of a step wedge should be obtained at 80 kilovolt peak. On the Fuji machine, the image should be obtained on a 14- $\times 17$-inch cassette set in fixed mode, S, 200; processed with GA, 1; GT, A; GS, $0 ; \mathrm{GC}, 1.0$; and RE, 0 . RN and RT can be any value. Adjust exposure (mAs) until the step wedge is well exposed with most levels visible. Record exposure factors $(\mathrm{kVp}, \mathrm{mAs}$, focal-spot film distance, focal-spot size). Make two such exposures. Print final images. Change to GA, 2; reprint new images. Reset image processing to GA, 1; RN, 5; RT, S; and RE, 10. Reprint new images. Store for comparison with later images.

Scan accuracy image. A scan accuracy image should be obtained. Place a steel ruler in the center of the imaging plate with edges parallel to the cassette hinges (perpendicular to the scan lines). Expose at $80 \mathrm{kVp}, 1 \mathrm{mR}$ approximate exposure. Record factors used. Process as organ name: sensitivity, organ code: 0900, semi mode, density: 1.0. Wait 10 minutes before processing IP. Store film for reference. Interpretation of this image is done by using a high-power magnifier $(8 x)$ to examine the edge of the steel ruler. The edge should appear straight.

Spatial resolution. Using a standard resolution phantom oriented obliquely to the edge of the cassette, obtain an image on a 14- $\times 17$-inch standard imaging plate at $80 \mathrm{kVp}$ at approximately $1 \mathrm{mR}$. Record exposure factors used. Process as organ name: sharpness, organ code: 0901, semi mode, density: 1.0. Store image for future reference. Repeat with a high-resolution imaging plate if used at your site.
$S$ number monitoring. Select a $14-\times 17$-inch imaging plate. Erase the plate. Use a flat-field exposure at $80 \mathrm{kVp}$ and approximately $1 \mathrm{mR}$. This should result in an $S$ of approximately 200 . This should be monitored weekly using the same IP and same exposure.

\section{ROUTINE OC PROCEDURES}

\section{Daily/Weekly}

Reject analysis. All rejected images should be inspected by the QC technologist as they are rejected (if possible) or at least daily. A proposed explanation for the image failure should be recorded for each image. The accumulated images over the preceding 4 weeks should be reviewed weekly looking for trends. Action should be taken as trends are detected.

\section{Weekly}

Check that the settings of image-processing algorithms have not changed. The commonly used algorithms should be checked weekly. The uncommonly used settings should be checked every 4 weeks (one-fourth done each week). Alternatively, if these factors could be stored on a memory card, one would simply reload the whole set of optimized factors each week without having to check for changes.

Using a standardized flat-field exposure, check the $\mathrm{S}$ number as described in "S number monitoring." Graph sequential results. Use control limits of \pm 60 units.

Repeat the standard step wedge image as described above and process it with the three indicated image-processing settings. Visually compare the new image with the original. If there is any visible change, measure to confirm the change. If greater than 0.25 OD units in any cell, investigate to find the cause.

Clean and inspect cassettes and imaging plates.

\section{Monthly}

Repeat the scan accuracy test as described above. Inspect both edges of ruler with $8 \times$ to $20 \times$ magnifier to look for unevenness of scan lines.

Repeat resolution test as described above in "spatial resolution." Compare resolution on the original and current images. 


\section{REFERENCES}

1. Seibert JA: Photostimulable phosphor system acceptance testing. AAPM Summer School, 1991, pp 899-929

2. Freedman M, Mun SK, Pe E, et al: Quality control of storage phosphor imaging devices, in Lemke HU, Inamura K, Jaffe CC, Felix R (eds): Computer Assisted Radiology. Berlin, Germany, Springer-Verlag, 1993, pp 456-460

3. Jafroudi H, Steller D, Freedman M: Computed Radiography Quality Control. Georgetown University Internal Procedure Document. Washington, DC, Georgetown University Medical School,

4. Freedman M, Mun SK, Pe E, et al: Image Optimization on the Fuji AC-1. SPIE Medical Imaging 1897:480-502, 1993
5. Nelson M, Pe E, Freedman M, et al: Image processing in musculoskeletal digital radiography: A comparison of the AGFA and FUJI bone algorithms. SPIE Medical Imaging 2167:854-867, 1994

6. Freedman M, Pe E, Lo S-CB, et al: Risk of unnecessary radiation overexposure of patients with a storage phosphor plate imaging system. SPIE Medical Imaging 1897:472-479, 1993

7. Busch HP, Georgi M (eds): Digital Radiography Workshop: Quality Assurance and Radiation Protection. Mannheim, Germany, May 7-9, 1992, pp 95-96

8. Busch HP, Georgi M (eds): Digital Radiography Workshop: Quality Assurance and Radiation Protection. Mannheim, Germany, May 7-9, 1992 